\title{
On the Validity of Modeling Rayleigh Scatterers by Spheroids
}

\author{
T. B. A. Senior and H. Weil \\ Electrical and Computer Engineering Department, University of Michigan, Ann Arbor, \\ MI 48109, USA
}

Received 8 April 1982/Accepted 23 June 1982

\begin{abstract}
For single homogeneous lossy dielectric Rayleigh particles, the manner in which the particle shape affects the absorption and scattering is examined by computing the polarizability tensor elements using a general purpose program valid for any rotationally symmetric body. The results for a number of generic shapes are compared with those for the corresponding spheroids. When the dielectric constant is real and negative, the resonances attributable to bulk and to shape-dependent or surface polariton modes are tracked as functions of the length-to-width ratio of the particle and the bulk dielectric constant. The adequacy of using spheroid approximations for individual particles in a dispersion is also discussed.
\end{abstract}

PACS: $78.30,41$

The scattering and absorption of electromagnetic radiation by dispersions of particles have been studied extensively using experimental, theoretical and numerical techniques. Nevertheless, because of difficulties inherent in all these approaches and the variety of physical parameters which influence the results, the search for valid simplifications is still of interest. One such simplification is to replace the particles by equivalent spheres, and because of the ease with which the Mie series expression for the field scattered by a sphere can be computed, this approximation is often employed. Its validity for highly non-spherical particles has been investigated by Holland and Gagne [1] who compared experimental data obtained using dispersions of flakes with Mie series results; and for particles which are much closer to spheres, a similar comparison was made by Wiscombe and Mugnai [2] using data computed with the Extended Boundary Condition (or T-matrix) method.

When the particles are small compared with the wavelength (Rayleigh scattering) a more general approximating shape which is often used is a spheroid, for which simple analytical expressions for the scattering and absorption are available. By considering spheroids ranging from thin needle-like shapes to lozenge-like disks, it has been shown [3-6] that the absorption can be strongly shape dependent, but the extent to which the conclusions are applicable to non-spheroids is not discussed. This is the topic that is addressed here by comparing the scattering and absorption for a number of generic shapes with those of the corresponding spheroids. The particles are assumed to be homogeneous, and the data for the individual particles are obtained using the program developed by Senior and Willis [7] for rotationally symmetric shapes.

The four shapes which are treated in most detail are circular cylinders with hemispherical or flat ends, and thick disks with semicircular (bevelled) or flat edges. These are all superficialiy similar to spheroids in being convex with longitudinal symmetry. In each case the criteria used for comparison are the values of the tensor elements $P_{11}$ and $P_{33}$ which are introduced in the following section, and which determine the scattering and absorption cross sections. The cases $\operatorname{Re}\{\varepsilon\}$ $\geqq 0$ and $\operatorname{Re}\{\varepsilon\}<0$ where $\varepsilon$ is the dielectric constant are treated separately.

\section{Tensor Elements and Their Computation}

For a homogeneous, nonmagnetic dielectric particle immersed in a homogeneous medium of permittivity $\varepsilon_{0}$ and irradiated by a plane linearly polarized elec- 
tromagnetic wave, the far zone scattered field at low frequencies is attributable to an induced electric dipole of moment $\overline{\mathbf{p}}=\varepsilon_{0} \overline{\overline{\mathbf{P}}} \cdot \hat{\mathbf{a}}$, where $\hat{\mathbf{a}}$ is a unit vector in the direction of the incident electric field and $\overline{\overline{\mathbf{P}}}$ is the electric polarizability tensor [8]. $\overline{\overline{\mathbf{P}}}$ is a symmetric tensor whose elements are functions only of the properties of the particle, i.e., its geometry and dielectric constant or permittivity $\varepsilon$ relative to that of the surrounding medium. The elements are complex if the material is lossy, but are otherwise real, and since the relative permeability is unity, there is no induced magnetic dipole.

In the special case of a particle with rotational symmetry about the $x_{3}$ axis of a Cartesian coordinate system $x_{1}, x_{2}, x_{3}$, the tensor diagonalizes with two of its diagonal elements equal, and

$\overline{\mathbf{p}}=\varepsilon_{0}\left[P_{11} \hat{\mathbf{a}}+\left(P_{33}-P_{11}\right)\left(\hat{\mathbf{a}} \cdot \hat{\mathbf{x}}_{3}\right) \hat{\mathbf{x}}_{3}\right]$.

The scattered electric field in the far zone is then

$\overline{\mathbf{E}}^{\mathrm{s}}(\overline{\mathbf{r}})=\frac{\mathrm{e}^{\mathrm{i} k r}}{k r} \overline{\mathbf{S}}(\hat{\mathbf{r}})$

with

$\overline{\mathbf{S}}(\hat{\mathbf{r}})=-\frac{k^{3}}{4 \pi}\left[P_{11} \hat{\mathbf{r}}_{\Lambda}\left(\hat{\mathbf{r}}_{\Lambda} \hat{\mathbf{a}}\right)+\left(P_{33}-P_{11}\right)\left(\hat{\mathbf{a}} \cdot \hat{\mathbf{x}}_{3}\right) \hat{\mathbf{r}}_{\Lambda}\left(\hat{\mathbf{r}}_{\Lambda} \hat{\mathbf{x}}_{3}\right)\right]$,

where $k$ is the propagation constant of the surrounding medium, $\hat{\mathbf{r}}$ is a unit vector in the direction of the point of observation, and a time factor $\exp (-\mathrm{i} \omega t)$ has been assumed and suppressed.

The differential scattering cross section is

$\sigma(\hat{\mathbf{r}})=4 \pi / k^{2} \overline{\mathbf{S}} \cdot \overline{\mathbf{S}}^{*}$,

where the asterisk denotes the complex conjugate, and by integrating over all angles, the total scattering cross section is found to be

$\sigma_{T}=\frac{k^{4}}{6 \pi}\left[\left|P_{11}\right|^{2}+\left(\left|P_{33}\right|^{2}-\left|P_{11}\right|^{2}\right)\left(\hat{\mathbf{a}} \cdot \hat{\mathbf{x}}_{3}\right)^{2}\right]$.

In the forward scattering direction, $\hat{\mathbf{r}} \cdot \hat{\mathbf{a}}=0 \mathrm{implying}$

$\hat{\mathbf{a}} \cdot \overline{\mathbf{S}}=\frac{k^{3}}{4 \pi}\left[P_{11}+\left(P_{33}-P_{11}\right)\left(\hat{\mathbf{a}} \cdot \hat{\mathbf{x}}_{3}\right)^{2}\right]$,

and from the forward scattering theorem [9] the extinction cross section is

$\sigma_{\mathrm{ext}}=k \operatorname{Im}\left\{P_{11}+\left(P_{33}-P_{11}\right)\left(\hat{\mathbf{a}} \cdot \hat{\mathbf{x}}_{3}\right)^{2}\right\}$.

For a lossy particle the absorption dominates the scattering at low frequencies and $\sigma_{\text {ext }} \simeq \sigma_{A}$, but in the absence of loss, $\sigma_{\text {ext }}=\sigma_{T}$ to the leading order in $k$.

The above quantities are all expressed in terms of the tensor elements $P_{11}$ representing the component of the dipole moment transverse to the axis of symmetry and $P_{33}$ representing the longitudinal component. From (4) and (5) we observe that if $\hat{\mathbf{a}} \cdot \hat{\mathbf{x}}_{3}=0$ or \pm 1 corresponding to an incident electric vector perpendicular or parallel to the axis respectively, $\sigma_{T}$ and $\sigma_{A}$ depend on a single tensor element alone, but for all other polarizations, both elements are involved. The elements themselves are expressible as weighted surface integrals of potential functions independent of the irradiation, and integral equations have been developed with which to compute the potentials [8]. The equations are relatively simple in the case of a rotationally symmetric body whose profile is defined as $\varrho=\varrho\left(x_{3}\right)$ where $\varrho$ and $x_{3}$ are cylindrical polar coordinates. If $s$ is the arclength along the profile, [Ref. 8, Eq. (10)] can be written as

$P_{11}=-\pi \int_{B} \varrho W_{1}(s) \cos \alpha d s$,

where the integration is over the entire profile and

$\alpha=\arctan \left(\partial \varrho / \partial x_{3}\right)$.

$W_{1}(s)$ is proportional to the surface value of a total potential and satisfies the integral equation

$$
\begin{aligned}
(1+\varepsilon) /(1-\varepsilon) W_{1}(s) & \\
= & 2 \varrho+\pi^{-1} \int_{B} W_{1}\left(s^{\prime}\right)\left\{\varrho \cos \alpha^{\prime} \Omega_{2}\right. \\
& \left.+\left[\left(x_{3}^{\prime}-x_{3}\right) \sin \alpha^{\prime}-\varrho^{\prime} \cos \alpha^{\prime}\right] \Omega_{1}\right\} \varrho^{\prime} d s^{\prime}
\end{aligned}
$$

which is deduced from [Ref. 8, Eq. (20)]. Similarly,

$P_{33}=2 \pi \int_{B} \varrho W_{3}(s) \sin \alpha d s$

with

$$
\begin{aligned}
& (1+\varepsilon) /(1-\varepsilon) W_{3}(s) \\
& =2 x_{3}+\pi^{-1} \int W_{3}\left(s^{\prime}\right)\left\{\varrho \cos \alpha^{\prime} \Omega_{1}\right. \\
& \left.\quad+\left[\left(x_{3}^{\prime}-x_{3}\right) \sin \alpha^{\prime}-\varrho^{\prime} \cos \alpha^{\prime}\right] \Omega_{0}\right\} \varrho^{\prime} d s .
\end{aligned}
$$

The quantities $\Omega_{m}(m=0,1,2)$ are defined in terms of the cylindrical coordinates of the two points $(\varrho, \phi, z)$ and $\left(\varrho^{\prime}, \phi^{\prime}, z^{\prime}\right)$ on the surface as

$\Omega_{m}=\int_{0}^{\pi} \frac{\cos ^{m} \psi d \psi}{R^{3}}$,

where $\psi=\phi-\phi^{\prime}$

$R^{2}=\varrho^{2}+\varrho^{\prime 2}-2 \varrho \varrho^{\prime} \cos \psi+\left(z-z^{\prime}\right)^{2}$,

and can be expressed as complete elliptic integrals. Since the eigenvalues of (7) and (9) are all real with moduli not less than unity [8], each integral equation has a unique solution if $\varepsilon$ is complex or $\varepsilon$ is real and positive.

For any rotationally symmetric body whose profile can be constructed from straight line and circular arc segments, the program DIELCOM developed by Senior and Willis [7] solves the equations by the 


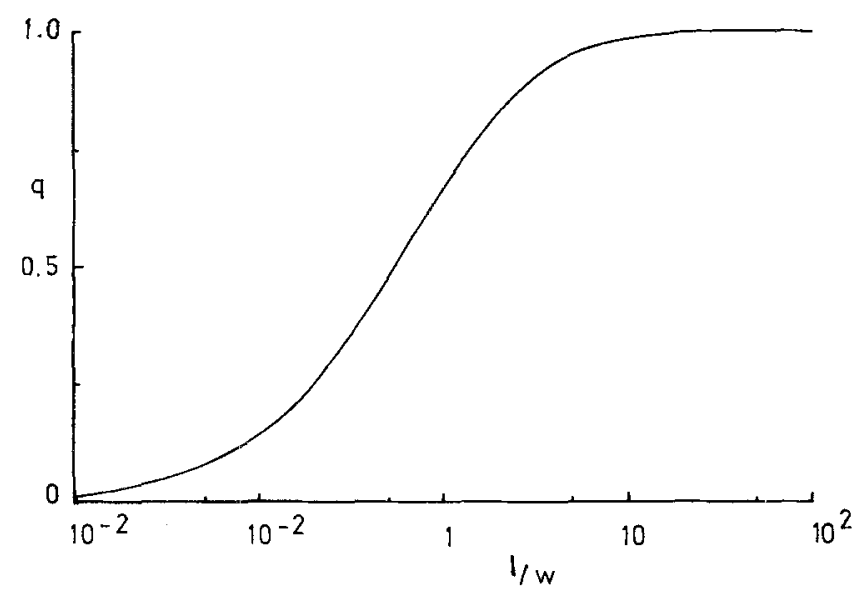

Fig. 1. Shape factor $q$ for spheroids as a function of the length-towidth ratio $l / w$

moment method and computes the normalized tensor elements $P_{11} / V$ and $P_{33} / V$, where $V$ is the volume of the body. Concave (re-entrant) shapes can be treated as well as multiply-connected ones, e.g., a torus. Bodies having two or more disjoint portions can also be considered provided their material parameters are the same and they have a common axis of symmetry. The program can therefore be used to examine the effect of particle shape on scattering and absorption. A similar program is available [10] for rectangular parallelepipeds.

\section{Results for $\operatorname{Re}\{\varepsilon\} \geqq 0$}

If $\operatorname{Re}\{\varepsilon\} \geqq 0$ the scattering and absorption are primarily bulk effects, and $P_{11}$ and $P_{33}$ are relatively insensitive to the precise details of the particle's shape. It is therefore to be expected that the values can be estimated using a particle of similar but simpler shape, and a convenient one to choose is a homogeneous spheroid for which analytical expressions for the tensor elements are available. For a spheroid of volume $V$

$P_{11}(\varepsilon)=V[q / 2+1 /(\varepsilon-1)]^{-1}$,

$P_{33}(\varepsilon)=V[(1-q)+1 /(\varepsilon-1)]^{-1}$,

where $q$ is a monotonic function of the length-to-width ratio $l / w$ (Fig. 1), increasing from zero in the disk limit of an oblate spheroid $(l / w=0)$, through $2 / 3$ for a sphere $(l / w=1)$, to unity for a needle-like prolate spheroid $(l / w$ $=\infty)[6]$.

For a convex and symmetric particle, it is found that a spheroid of the same volume and length-to-width ratio does indeed provide an excellent approximation to both the real and imaginary parts of $P_{11}$ and $P_{33}$. In the case of a right circular cylinder with hemispherical end caps (Fig. 2a) and length-to-width ratio of 5 for $\varepsilon=\gamma$ and $\varepsilon=\gamma(1+\mathrm{i} 0.5), 0 \leqq \gamma \leqq 10$, Fig. 3 , shows the

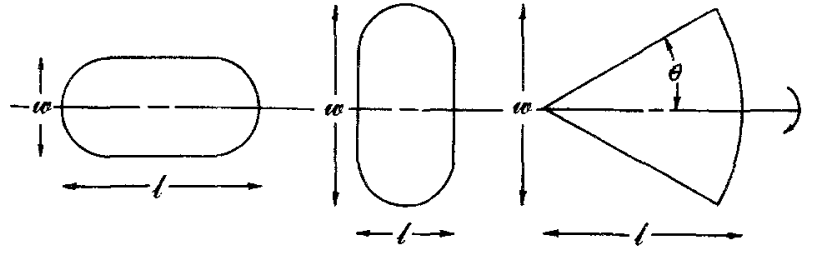

(a)

(b)

(c)

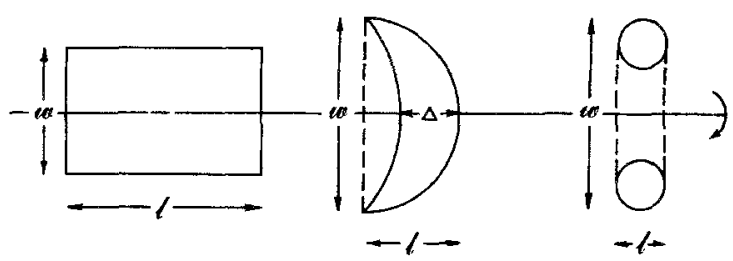

(d)

(e)

$(f)$

Fig. $2 a-f$. Selected bodies of revolution about the $x_{3}$ axis. (a) is a hemispherically-capped cylinder, for which $l / w \geqq 1$; the analogous body having $l / w \leqq 1$ is the bevelled disk shown in (b). (c) is a roundbacked cone of half angle, $\theta$, for which $l / w \geqq 0.5$ if $\theta \leqq \pi / 2$. (d) is a right circular cylinder. (e) is a scooped-out hemisphere for which $l / w$ $=0.5$ independently of $\Delta$, and (f) is a torus

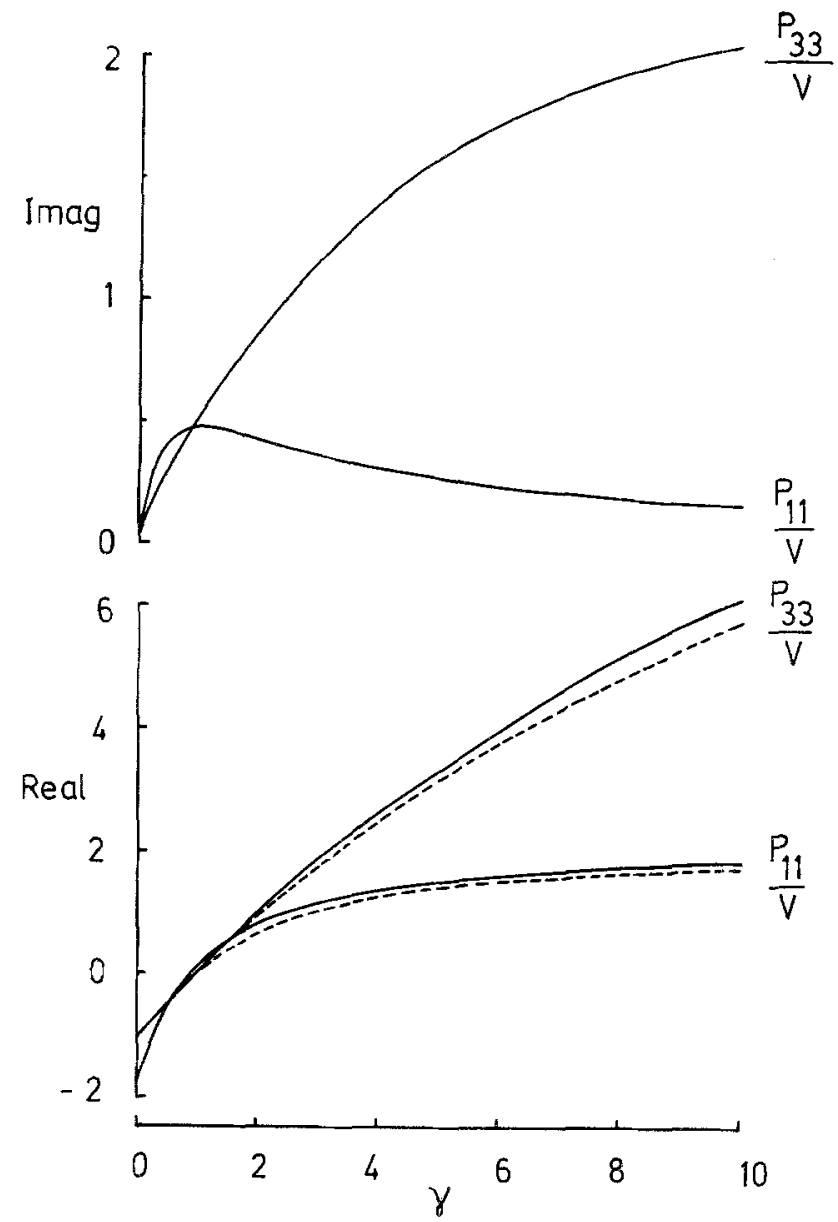

Fig. 3. The real and imaginary parts of $P_{11} / V$ and $P_{33} / V$ for a hemispherically-capped cylinder of length-to-width ratio 5 for $\varepsilon=\gamma$ $(--)$ and $\varepsilon=\gamma(1+\mathrm{i} 0.5)(-)$ 

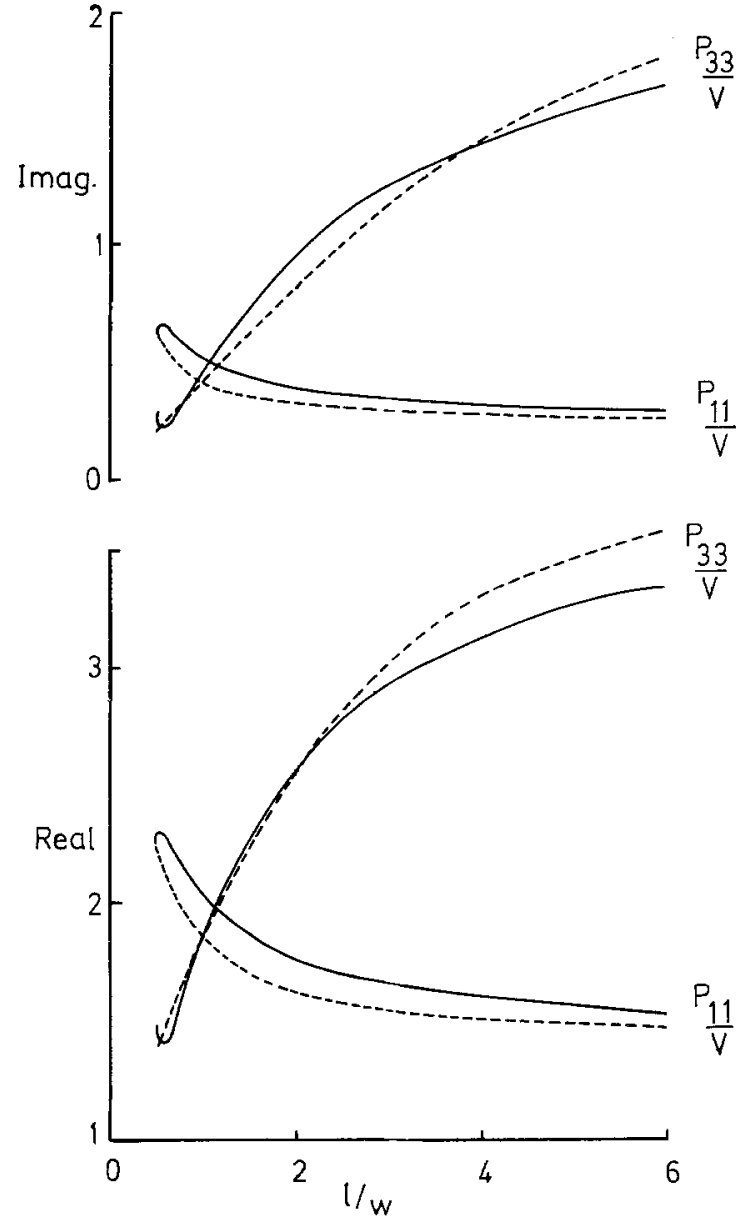

Fig. 4. The real and imaginary parts of $P_{11} / V$ and $P_{33} / V$ for a roundbacked cone of length-to-width ratio $l / w$ for $\varepsilon=5+\mathrm{i} 2.5(-)$ compared with the corresponding spheroid values (-- )

Table 1. Scooped-out hemisphere (Fig. 2e)

\begin{tabular}{lllll}
\hline$\Delta / l$ & $\operatorname{Re}\left\{P_{11} / V\right\}$ & $\operatorname{Im}\left\{P_{11} / V\right\}$ & $\operatorname{Re}\left\{P_{33} / V\right\}$ & $\operatorname{Im}\left\{P_{33} / V\right\}$ \\
\hline 1.0 & 2.24 & 0.623 & 1.48 & 0.267 \\
0.8 & 2.35 & 0.709 & 1.49 & 0.283 \\
0.6 & 2.48 & 0.827 & 1.57 & 0.332 \\
0.4 & 2.65 & 1.00 & 1.70 & 0.432 \\
0.2 & 2.87 & 1.36 & 1.99 & 0.788 \\
\hline
\end{tabular}

values of $P_{11} / V$ and $P_{33} / V$ computed from (6) through (9). The analogous results for a spheroid differ by less than (about) five percent, and comparable agreement has been found for other values of $l / w$ and $\varepsilon$ for which the comparison has been made.

If the particle is convex but asymmetric, the corresponding spheroid may still provide a reasonable estimate of the tensor elements, but the accuracy is less. This is illustrated in Fig. 4 where the real and imaginary parts of $P_{11} / V$ and $P_{33} / V$ for round-backed cones (Fig. 2c) of increasing half angle $\theta$ are compared with the values for spheroids having the same $l / w(=1 / 2 \operatorname{cosec} \theta)$. The relative permittivity is $\varepsilon=5(1+i 0.5)$ and the extreme case $\theta=\pi / 2$ corresponds to a hemisphere. We observe that the spheroid data exhibit the required variation as functions of $l / w$, but the discrepancies are now as much as $20 \%$. Nevertheless, the agreement is better than might have been expected in view of the differences in shape of the two bodies. We also remark that the rapid changes in the curves for the cone near $l / w=0.5$ are real, and come about because of the virtual insensitivity of $l / w$ to $\theta$ as $\theta$ approaches $\pi / 2$.

For a concave (re-entrant) shape it is not surprising that a spheroid is generally inadequate for estimating the tensor elements, not least because the parameter $l / w$ does not then reflect the presence of the concavity. Table 1 lists data for a sequence of "scooped-out" hemispheres (Fig. 2e) having $\varepsilon=5(1+\mathrm{i} 0.5)$. As the shell thickness and, hence, $\Delta / l$ decrease, the normalized elements increase monotonically, implying increased scattering and absorption per unit volume of the material. This is in accordance with previous observations [6] for thin spheroids (disks and needles), but in the present instance each body has a length-to-width ratio of 0.5 independently of $\Delta$. For the corresponding spheroid $P_{11} / V=2.240+\mathrm{i} 0.6046$ and $P_{33} / V=1.380$ $+\mathrm{i} 0.2193$, and, as $\Delta / /$ decreases, the values of $\operatorname{Im}\left\{P_{11} / V\right\}$ and $\operatorname{Im}\left\{P_{33} / V\right\}$ in particular depart radically from those for the spheroid.

A similar conclusion obtains in the case of tori (Fig. 2f) for which the predicted values of $P_{11} / V$ and $P_{33} / V$ based on the corresponding (oblate) spheroids are in error by as much as an order of magnitude or more. For these bodies a spheroid having the same length-towidth ratio cannot be used for estimating the tensor elements per unit volume and they will not be considered in the next section in which $\operatorname{Re}\{\varepsilon\}<0$.

\section{The Region where $\operatorname{Re}\{\varepsilon\}<0$}

This region is characterized by narrow ranges of $\varepsilon$ where the absorption can be large, and the location of these, as well as the absorptivity, are influenced by the shape of the particle. It is therefore expected that the accuracy with which a spheroid models the tensor elements will also be affected, but before presenting our results, it may be helpful to discuss the underlying physics.

The manner in which the location of the absorption peaks in the complex $\varepsilon$ plane depends on the particle shape has been extensively studied from the point of view of polariton modes. These are associated with hybrid photon-optical phonon quanta, i.e., polaritons, and express the coupling of the incident photon ra- 
diation to the vibrational modes of the crystal material, in particular, to the transverse optical modes, which involve the relative motion of atoms and molecules within the unit crystal cell. Shape effects are important in determining the standing-wave modes. A shape dependence of the absorption comes about when significant electromagnetic-vibrational coupling is associated with standing waves which decay towards the interior of the particle, and the coupling is then said to be in a surface polariton mode. In addition, there may be volume dependent absorption attributable to any uniform mode that is excited.

The basic physics of polariton modes for infinite media was discussed by Nelson [11], and the way in which a particle's shape affects the absorption has been investigated by a number of authors. As a practical matter, the study of shape effects comes down to the solution of an electromagnetic scattering and absorption problem for particles composed of a material whose bulk dispersion relation can be obtained from the classical equations for the vibrational motion of the crystal lattice, or from the quantum mechanical equivalent. The Mie series solutions for spherical and (infinite) cylindrical particles have been examined in this context by Englman and Ruppin $[12,13]$. The leading (dipole) terms are the dominant ones for the electrically small particles of interest to us here. For parallelepipeds (including the cube) as well as spheres the polariton modes were determined by Langbein [14] using numerical integration to obtain the coefficients in a spherical harmonic expansion of the fields. Extensive symmetry arguments were employed to aid in locating the values of $\varepsilon$ at which significant absorption could occur. A cube has also been treated by Fuchs [15, 16] and Ruppin [17] by solving numerically the integral equations for the fields, and Huffman and Bohren [5] have studied Rayleigh scattering by ellipsoids.

When the problem is formulated as a classical Rayleigh scattering problem for a particle with a given $\varepsilon=\varepsilon(\omega)$ where $\omega$ is the radian frequency, the techniques described in Sect. 1 can be used to locate the resonances and compute the absorption due to the polariton modes. The resonances occur at the negative real values of $\varepsilon$ corresponding to the eigenvalues of (7) and (9), or equivalently, at the poles of the matrix elements $P_{11}$ and $P_{33}$. In the particular case of a spheroid, these poles can be obtained analytically. From (10), $P_{11}$ has only a single pole (resonance) at

$\varepsilon \equiv \varepsilon_{11}=1-2 / q$,

and from (11), there is a single pole for $P_{33}$ at

$\varepsilon \equiv \varepsilon_{33}=1-1 /(1-q)$.

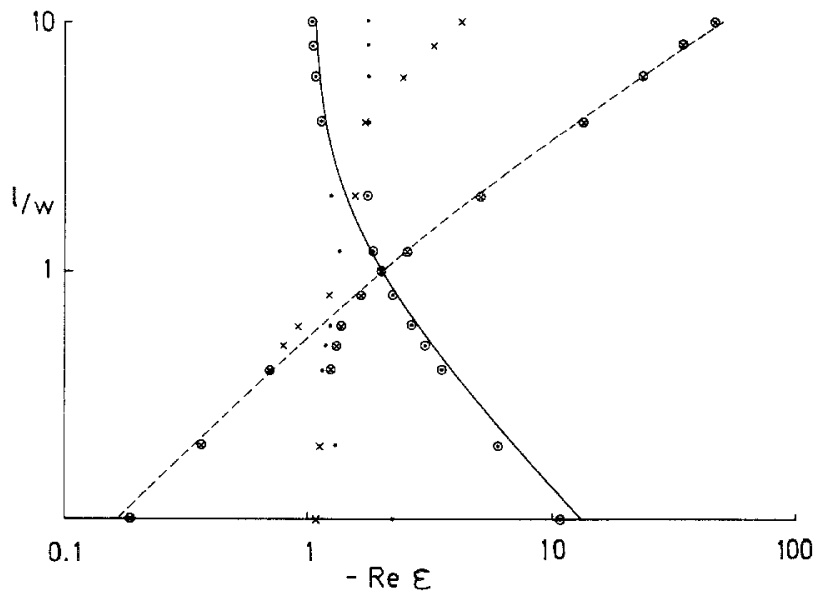

Fig. 5. The locations of the spheroid resonances $\left(P_{11}-\ldots, P_{33}---\right)$ compared with those for hemispherically-capped cylinders $(l / w \geqq 1)$ and bevelled disks $(l / w \leqq 1)$. The dots are for $P_{11}$ resonances, the crosses are for $P_{33}$ resonances, and the locations of the dominant resonances are circled

For a sphere $\varepsilon_{11}=\varepsilon_{33}=-2$, and using Fig. 1, it is a trivial matter to determine the poles for any length-towidth ratio $l / w$.

For other rotationally symmetric bodies, DIELCOM can be used to compute $P_{11}$ and $P_{33}$ as functions of $\varepsilon$, and thereby locate the values of $\varepsilon$ at which resonance occurs. When (7) or (9) is discretized as required by the moment method of solution used in DIELCOM, an $N \times N$ matrix is produced whose size is equal to the number $N$ of sampling points employed. The $N$ eigenvalues of the matrix are all real, and a subset of these are approximations to the eigenvalues of the corresponding integral equation. The others are a consequence of the discretization and, for the most part, tend to cluster in the vicinity of $\varepsilon=-1$. (Similar extraneous eigenvalues occur in $[14,16]$.) By computing the eigenvalues of the matrix for $N$ sufficiently large to provide an accurate determination of $P_{11}$ (or $\left.P_{33}\right)$, and then testing each to see whether it gives rise to a local maximum in the imaginary part of the tensor element, the eigenvalues of the integral equation itself as well as the strength of the associated resonances can be found.

\section{Results for $\operatorname{Re}\{\varepsilon\} \leqq 0$}

Using the above technique, data have been obtained for the shapes shown in Fig. 2a, b, and d. In the case of a spheroid each tensor element $P_{11}$ or $P_{33}$ has just a single resonance occurring for a negative real $\varepsilon$ whose value depends on $l / w$. The loci of these resonances in the $l / w$ vs $\operatorname{Re}\{\varepsilon\}$ plane for $0.1 \leqq l / 2 \leqq 10$ are shown by the solid and dashed lines in, for example, Fig. 5. 


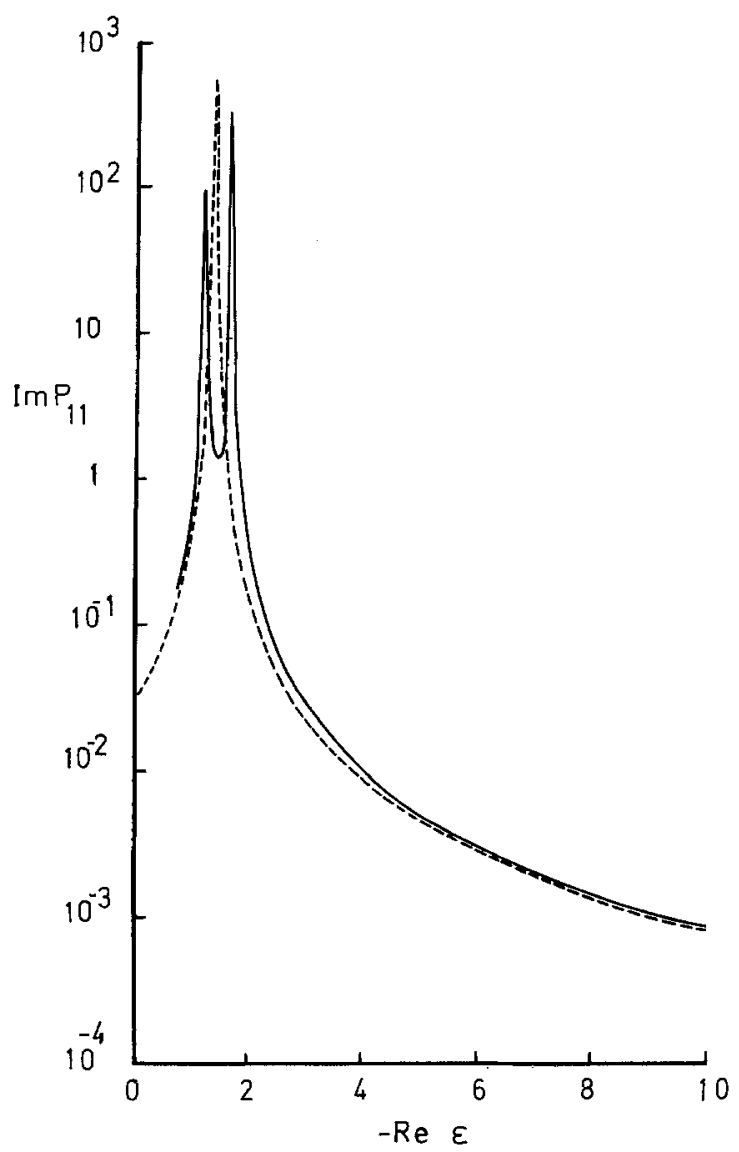

Fig. 6. The imaginary part of $P_{11}$ for a 2:1 hemispherically-capped cylinder $(-)$ and a $2: 1$ spheroid $(---)$ for $0 \geqq \operatorname{Re}\{\varepsilon\} \geqq-10$ with $\operatorname{Im}\{\varepsilon\}=0.01$

The hemispherically-capped cylinder $(l / w \geqq 1)$ and the bevelled disk $(l / w \leqq 1)$ are superficially similar to spheroids, prolate and oblate, and share the common limit of a sphere when $l / w=1$. In contrast to the spheroid, however, these bodies have tensor elements with more than one resonance, and the resonances computed for a range of length-to-width ratios are included in Fig. 5. Taking, for example, the case $l / w=10$, one observes that $P_{11}$ has a resonance at almost the same value of $\varepsilon$ as the corresponding spheroid, but there is in addition a weaker resonance at $\varepsilon=-1.73$. Similarly, for $P_{33}$, whose subdominant resonance occurs at $\varepsilon=-4.22$. As $l / w$ decreases, the resonances shift in location and their strengths change. Indeed, by the time $l / w=2$ is reached, the previously dominant resonance of $P_{11}$ has so weakened as to become the subdominant one, and has disappeared completely when $l / w=1$. The subdominant resonance of $P_{33}$ remains subdominant throughout and it also disappears as $l / w \rightarrow 1$. As $l / w$ decreases below unity, a subdominant resonance of each of $P_{11}$ and $P_{33}$ reappears. In the case of $P_{11}$, the dominant resonance occurs close to the spheroid one and remains do-

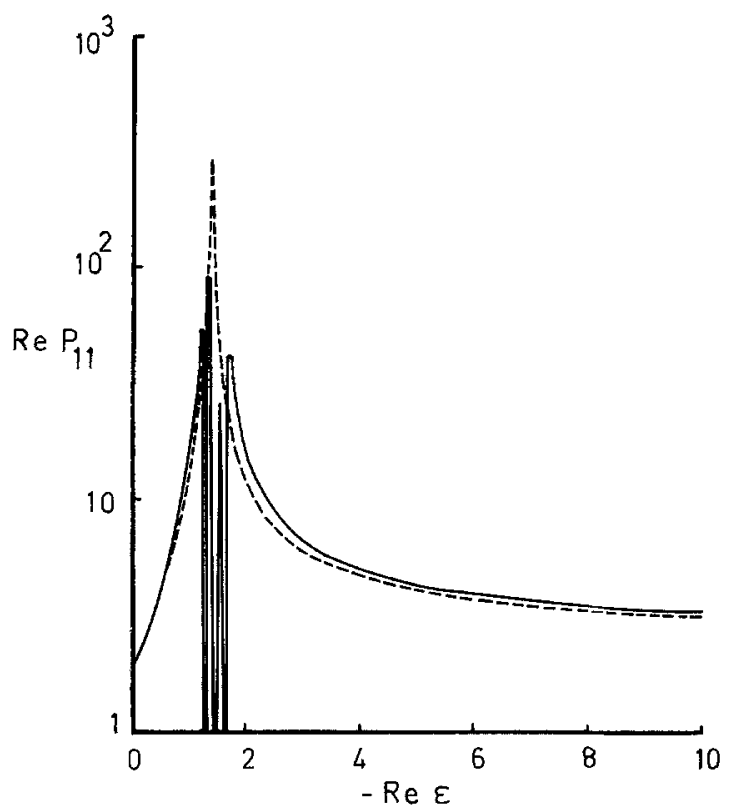

Fig. 7. The magnitude of the real part of $P_{11}$ for a $2: 1$ hemispherically-capped cylinder (- - ) and a $2: 1$ spheroid (-- ) for $0 \geqq \operatorname{Re}\{\varepsilon\} \geqq-10$ with $\operatorname{Im}\{\varepsilon\}=0.01$

minant throughout. For $P_{33}$, however, the previously dominant resonance progressively weakens, and for $l / w \lesssim 0.5$ the main resonance is also close to the spheroid one.

To better appreciate the significance of these results, Figs. 6 through 9 show the magnitudes of the real and imaginary parts of $P_{11}$ and $P_{33}$ for $l / w=2$ as functions of $\varepsilon$ with $0 \geqq \operatorname{Re}\{\varepsilon\} \geqq-10$ and $\operatorname{Im}\{\varepsilon\}=0.01$ (to ensure finite values at resonance). The curves for the corresponding spheroids are also shown. The overwhelming impression is the close agreement between the data for the two bodies for all values of $\varepsilon$ with the possible exception of those in the immediate vicinity of a resonance. We remark that the complex character of the cylinder curve in Fig. 7 is a consequence of plotting on a logarithmic scale the modulus of a typical resonance curve when two resonances are close together. Figure 8 also shows the genesis of a third resonance close to the subdominant one, and this is actually present for most $l / w>1$.

For a right circular cylinder with flat ends (Fig. 2d) the results analogous to those in Fig. 5 are shown in Fig. 10. The behavior is more complicated, and it is even possible that some of the lesser resonances have not been picked up. To judge from Fig. 10, each tensor element now has up to four discernible resonances, but for $l / w \gg 1$ the dominant resonance of $P_{33}$ far exceeds all others in magnitude. Conversely, for $l / w \ll 1$ the main resonance of $P_{11}$ dominates. Apart from $l / w$ in the range $0.3 \lesssim l / w \lesssim 3$, the dominant resonance for each tensor element occurs at a similar value of $\varepsilon$ as 


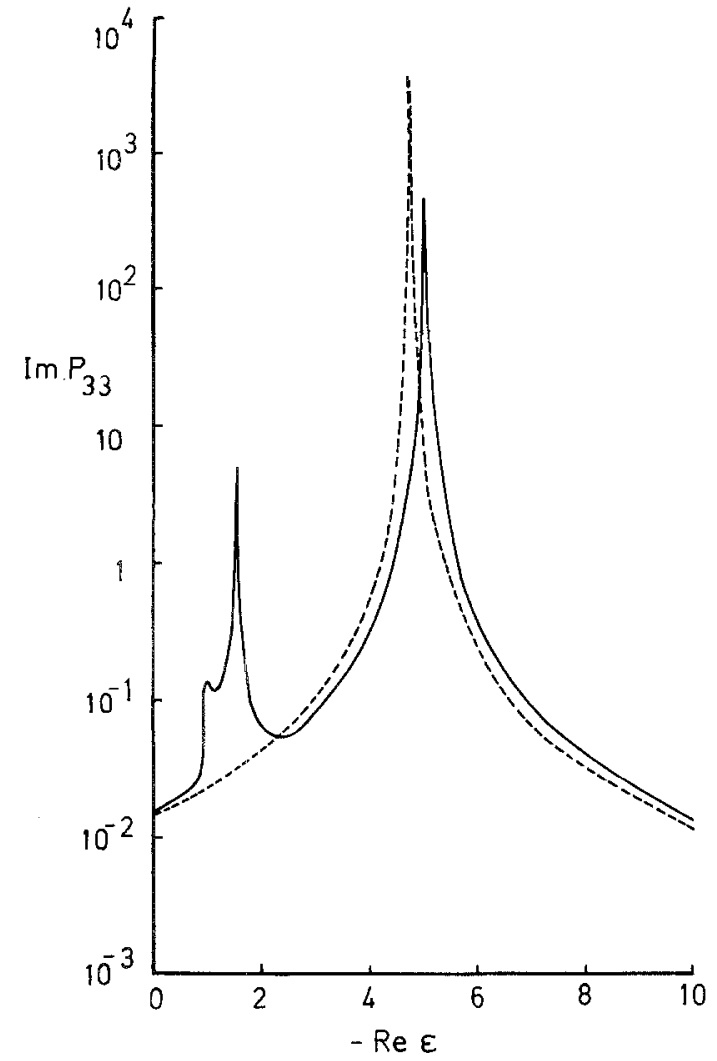

Fig. 8. The imagainary part of $P_{33}$ for a 2:1 hemispherically-capped cylinder (-) and a $2: 1$ spheroid $(--)$ for $0 \geqq \operatorname{Re}\{\varepsilon\} \geqq-10$ with $\operatorname{Im}\{\varepsilon\}=0.01$

that for the corresponding spheroid, and the loci of the spheroid resonances roughly delimit the loci for the cylinder. When $l / w \simeq 1$ the number of resonances is a maximum (four for each element), and the corresponding spheroid (i.e., a sphere) is least applicable as a guide. Even here, however, the failure may not, in practice, be as great as Fig. 10 would seem to indicate. For any given $l / w$ the resonances shown can differ by several orders of magnitude, and thus, for $P_{33}$ with $l / w=1$, there is a variation of two orders of magnitude between the most and least dominant.

\section{Conclusions}

For homogeneous particles which are small compared with the incident wavelength, program DIELCOM [7] can be used to compute the scattering and absorption of any rotationally symmetric shape. With its aid, the accuracy with which a spheroid models the scattering and absorption of other rotationally symmetric bodies having the same volume and length-to-width ratio $l / w$ has been examined. The physics of the problem naturally separate the investigation into two parts depending on whether $\operatorname{Re}\{\varepsilon\}$ is positive or negative. The

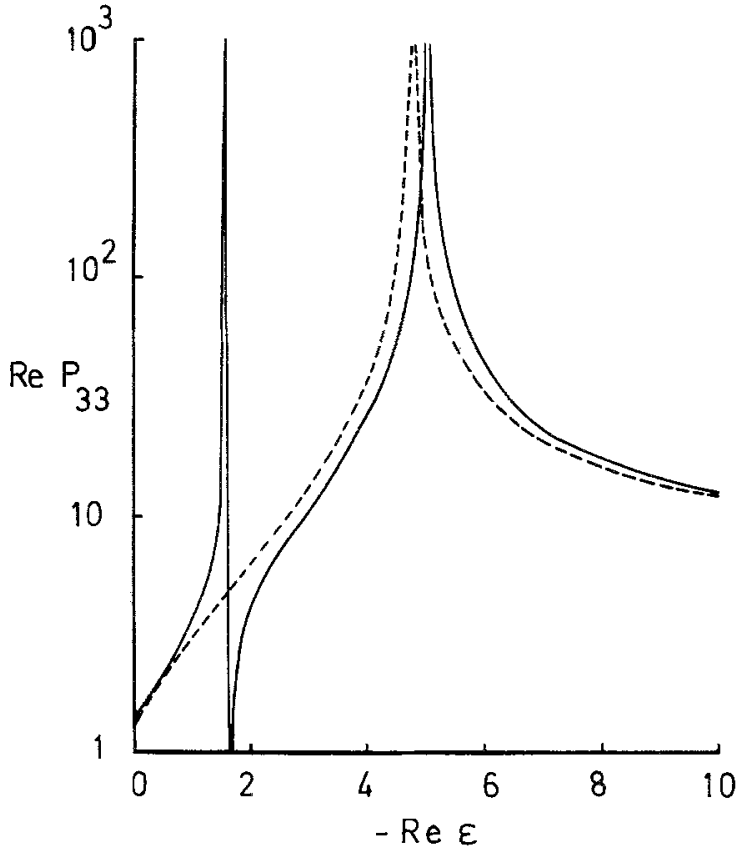

Fig. 9. The magnitude of the real part of $P_{33}$ for a $2: 1$ hemispherically-capped cylinder (-) and a $2: 1$ spheroid (-- ) for $0 \geqq \operatorname{Re}\{\varepsilon\} \geqq-10$ with $\operatorname{Im}\{\varepsilon\}=0.01$

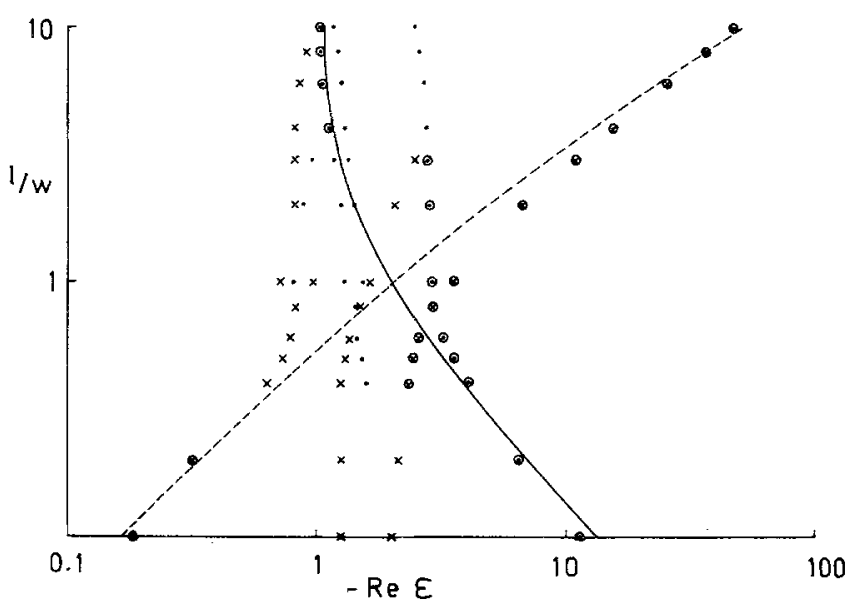

Fig. 10. The locations of the spheroid resonances $\left(P_{11}-, P_{33}\right.$ --- ) compared with those for right circular cylinders (Fig. 2d). The dots are for $P_{11}$ resonances, the crosses are for $P_{33}$ resonances, and the locations of the dominant resonances are circled

latter case occurs near resonances in $\varepsilon$ for the bulk material, and these give rise to peaks in the absorption spectra due to the material alone. The effects of the particle shape combine with the bulk effects to modify these peaks and also to create additional ones. In contrast, for $\operatorname{Re}\{\varepsilon\}>0$ there are no such absorption peaks.

The conclusions for the simpler case $\operatorname{Re}\{\varepsilon\}>0$ are readily summarized and are not at all surprising. To determine both the scattering and the absorption, a spheroid of the same length-to-width ratio is an excel- 
lent model if the particle is convex and symmetric in the axial direction. For a particle which is convex but not longitudinally symmetric, the spheroid can still give results which are accurate enough for some practical purposes, but for concave (re-entrant) or multiply-connected shapes such as a scooped-out hemisphere or torus which do not even superficially resemble a spheroid, the spheroid having the same $l / w$ is generally inadequate.

When $\operatorname{Re}\{\varepsilon\}<0$ only the convex symmetrical shapes shown in Fig. 2a, b, and d were explored numerically. The results are consistent with the following physical picture. Each tensor element for a spheroid has only a single resonance, and since the internal (static) field is uniform, the resonance is a bulk effect in which the absorption is proportional to the volume. Shapes other than a spheroid display more than one resonance, and the internal field has additional non-uniform components which increase with increasing departure from a spheroid. Near resonance these components decay with distance from the surface and can be interpreted as surface polariton modes. The resulting absorption then involves a volume of material which is less than the volume of the particle, leading to resonances which are in general weaker than that associated with a bulk mode.

To eliminate the rapid variation of $\operatorname{Im}\{\varepsilon\}$ with $\operatorname{Re}\{\varepsilon\}$ which is characteristic of the polariton radiationvibration interaction in the bulk material, and thereby focus on the shape effects alone, the computations were carried out for a range of $l / w$ by varying $\operatorname{Re}\{\varepsilon\}$ with $\operatorname{Im}\{\varepsilon\}$ fixed. For cylinders and disks with curved and flat ends, the results show that if $l / w$ is well away from unity the dominant absorption peak is that associated with the bulk mode, and this is close to the peak of the corresponding spheroid. As $l / w$ approaches unity, other absorption peaks associated with the surface polariton modes become more significant. The number of such peaks is greater when the ends of the body are flat rather than curved, i.e., when the body departs more from the smooth spheroidal shape. Thus, for $0.3 \leqslant l / w \lesssim 3$, the resonance behavior may differ substantially from that of a spheroid, but this range apart, the resonances are roughly delimited by the loci of the spheroidal peaks. Beyond these bounds on $\operatorname{Re}\{\varepsilon\}$, the spheroid again provides a good fit to both the scattering and the absorption.
The conclusions drawn here concerning the modeling of individual particles by spheroids can be extended to the more practical problem of the validity of such modeling for dispersion of particles. In particular, if the dispersion is sufficiently tenuous to permit the single scattering approximation, the effect of a probabilistic distribution of particle orientations can be accounted for in (4) and (5) by replacing $(\hat{\mathbf{a}} \cdot \hat{\mathbf{x}})^{2}$ by its average value. Multiplication by the mean number density of particles will then convert (4) and (5) to cross sections per unit volume of the dispersion. Hence, if the $P_{11}$ and $P_{33}$ values for the individual particle shapes correspond closely to those of a spheroid model, the scattering and absorption of the dispersion of particles will be well modeled by a similar dispersion of spheroids. The converse is also true, and averaging over particle orientations cannot be relied upon to blur out the effects of poor modeling of the individual particles by spheroids.

Acknowledgements. This work was supported by the U.S. Air Force Office of Scientific Research Grant 82-0112 and by the U.S. Army Armament Research and Development Command, Chemical Systems Laboratory, under Contract DAAK11-81-K-0004. The authors are indebted to D. Ksienski and T. M. Willis III for their assistance with the computations.

\section{References}

1. A.C.Holland, G.Gagne: Appl. Opt. 9, 1113-1121 (1970)

2. W.Wiscombe, A.Mugnai: In Light Scattering by Irregular Particles, ed. by D.W.Schuerman (Plenum Press, New York 1980) pp. 141-152

3. J.M.Greenberg: Loc. cit., pp. 7-24

4. J.Embury: Loc. cit., pp. $97-102$

5. D. R. Huffman, C.F.Bohren: Loc. cit., pp. 103-111

6. T.B.A.Senior: Appl. Opt. 19, 2483-2485 (1980)

7. T.B.A.Senior, T.M.Willis III: Rayleigh Scattering by Dielectric Bodies. IEEE Trans. AP-30, (November 1982)

8. T.B.A.Senior: Radio Sci. 11, 477-482 (1976)

9. M. Born, E.Wolf: Principles of Optics, 2nd ed. (Pergamon Press, Oxford 1964) p. 658

10. D.F.Herrick, T.B.A. Senior: Appl. Phys. 13, 175-183 (1977)

11. D.F.Nelson: Electric, Optic, and Acoustic Interaction in Dielectrics (Wiley, New York 1979) pp. 184-192

12. R. Englman, R. Ruppin, J. Phys. (C) (Proc. Phys. Soc.) 1, 614-643 (1968)

13. R. Ruppin, R.Englman: Rep. Prog. Phys. 33, 149-196 (1970)

14. D.Langbein: J. Phys. A 9, 627-644 (1976)

15. R.Fuchs: Phys. Rev. B 11, 1732-1740 (1975)

16. R. Fuchs: Phys. Rev. B 18, 7160-7162 (1978)

17. R. Ruppin: Opt. Commun. 26, 360-362 (1978) 